\title{
Shape Limit in Triangular Spiral Tilings
}

\author{
T. Sushida, A. Hizume And Y. Yamagishi* \\ Department of Applied Mathematics and Informatics, Ryukoku University, Seta, Otsu, 520-2194 Japan \\ Phyllotaxis is the study of arrangements of leafs and florets. The topology of triangular spiral (multiple) tilings \\ with opposed parastichy pairs is intimately related to the phyllotaxis theory and continued fractions. It is shown \\ that, if the divergence angle of the genetic spiral is given as a quadratic irrational and fixed, then the limit set of \\ the shape parameters of triangular tiles, as the parastichy numbers tend to infinity, is a finite set. In particular, \\ the limit is the golden section if the divergence angle is 'ultimately golden'.
}

DOI: 10.12693 /APhysPolA.126.633

PACS: 02.10.De, 02.30.Oz, 02.40.Pc, 87.10.Hk

\section{Introduction}

Phyllotaxis [1] is an interdisciplinary subject related to physics, biology, and mathematics [2, 3], where the golden section $\tau=(1+\sqrt{5}) / 2$, the Fibonacci numbers, and continued fraction expansions play important roles $[4,5]$. In [6], a quasi-crystalline structure is observed in a circular defect line in the parastichy transition of spiral phyllotaxis. Recently, there are intensive studies on the dynamical models that generate spiral phyllotaxic patterns [7-10], as well as geometric approaches on the spiral phyllotaxis [11-13].

\section{(a)}
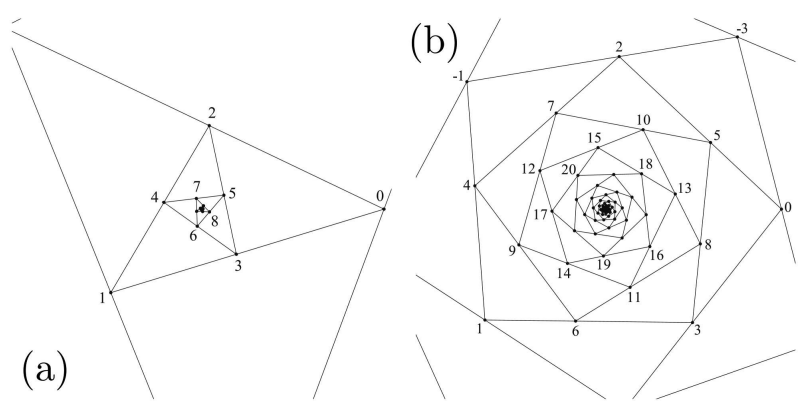

Fig. 1. Triangular spiral tilings with vertex sets $\left\{z^{j}\right\}_{j \in \mathbb{Z}}, z=r \mathrm{e}^{2 \pi \mathrm{i} \tau}, \tau=(1+\sqrt{5}) / 2$. The number $j$ denotes the point $z^{j} \in \mathbb{C}$. (a) $r=0.6780$, opposed parastichy pair $\{2,3\}$. (b) $r=0.9328$, opposed parastichy pair $\{5,3\}$.

One of the most simplified mathematical models of spiral phyllotaxis is the triangular spiral tiling that admits transitive action of a similarity transformation group, with no rotational symmetry, Fig. 1 . In the previous paper [14], we have shown that the parameter space $P_{v}$ of the generators $z=r \mathrm{e}^{\mathrm{i} \theta}$ of triangular spiral (multiple) tilings of multiplicity $|v| \neq 0$ with opposed parastichy pairs is a nowhere dense subset of the unit disk

*corresponding author; e-mail: yg@rins.ryukoku.ac.jp

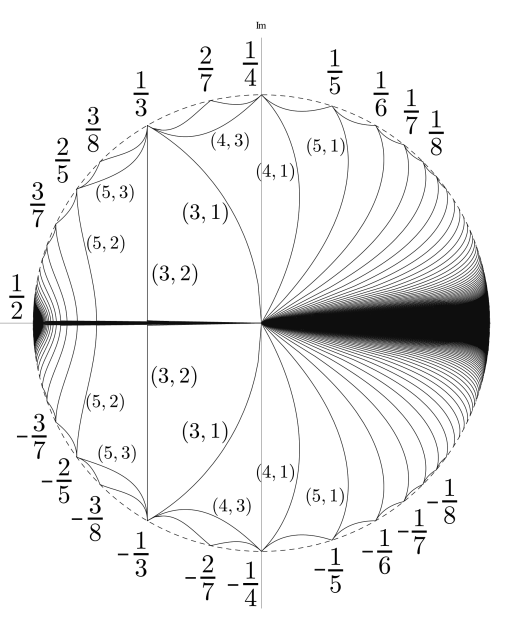

Fig. 2. The set $P_{1} \cup P_{-1}$ of generators for triangular spiral tilings with opposed parastichy pairs. The $\operatorname{arcs} P_{m, n, 1}$ and $P_{m, n,-1}$, defined in [14], are denoted by $(m, n)$.

$\mathbb{D}$, which is a countable family of real algebraic curves parametrized by the divergence angle $\theta$, see Fig. 2, whereas the parameter space $Q_{v}$ for triangular spiral (multiple) tilings with non-opposed parastichy pairs is a dense subset of $\mathbb{D}$, being a countable union of real algebraic curves parametrized by the plastochrone ratio $1 / r$. In $P_{v}$, the opposed parastichy pair is described by the continued fraction expansion of $\theta / 2 \pi$. The union $\cup_{v} P_{v}$ is a dense subset of $\mathbb{D}$.

In this paper we describe the relationship between the continued fraction of the divergence angle and the triangular spiral (multiple) tilings with an opposed parastichy pairs, and study the limit set $\Omega(\theta)$ of the 'shape parameters' of tiles, as $r \rightarrow 1$, of triangular spiral multiple tilings with opposed parastichy pairs. It is shown that if $\theta / 2 \pi$ is a quadratic irrational, then $\Omega(\theta)$ is a finite set of quadratic irrationals. It is known that most divergence angles $\theta$ of the spirals observed in plant phyllotaxis are written in the form $\theta=2 \pi(a \tau+b) /(c \tau+d)$, for some $a, b, c, d \in \mathbb{Z}$ with $a d-b c=1$ [5]. If it is the case, then we have $\Omega(\theta)=\{-\tau,-1 / \tau\}$. 


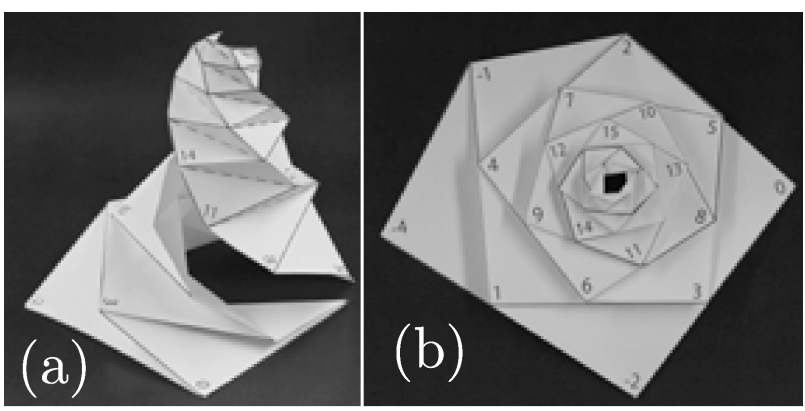

Fig. 3. Paper-folding for the tiling Fig. 1b. (a)

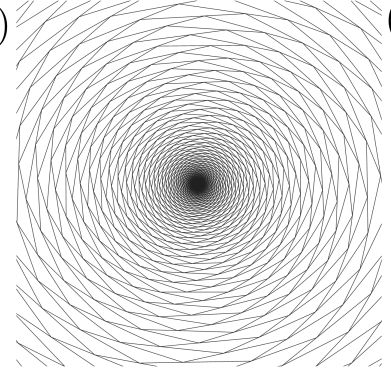

(b)

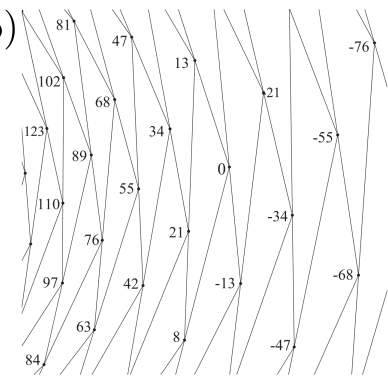

Fig. 4. Triangular spiral tiling generated by $(0.9965) \exp (2 \pi \mathrm{i} \cdot \tau), \tau=(1+\sqrt{5}) / 2$, with an opposed parastichy pair $\{8,13\}$. (a) Global view around the origin. (b) Local view around the tile $T_{0}$.

In the International Conference of Quasicrystals, Krakow, September 2013, we presented paper-folding sheets that build spiral towers whose top-down views are triangular tilings, Fig. 3. See $[14,15]$ for some backgrounds in figurative arts as applications of phyllotaxis and quasicrystals.

\section{Triangular spiral tilings and continued fractions}

For $x \in \mathbb{R}$, let

$$
x=a_{0}+\frac{1}{a_{1}+\frac{1}{a_{2}+\ldots}}=\left[a_{0} ; a_{1}, a_{2}, \ldots\right]
$$

be its continued fraction expansion [16, 17], where $a_{0} \in \mathbb{Z}, a_{i} \in \mathbb{N}, i \geq 1$. Define the sequences $\left\{p_{j}\right\}_{j \geq-1}$ and $\left\{q_{j}\right\}_{j \geq-1}$ by $p_{-1}=1, p_{0}=a_{0}, p_{1}=a_{0} a_{1}+1$, $p_{j+1}=a_{j+1} p_{j}+p_{j-1}, j \geq 1 ; q_{-1}=0, q_{0}=1, q_{1}=a_{1}$, $q_{j+1}=a_{j+1} q_{j}+q_{j-1}, j \geq 1$. In this paper we denote by $p_{j, k}=k p_{j}+p_{j-1}$, and $q_{j, k}=k q_{j}+q_{j-1}$ for $j \geq 0$, $0 \leq k \leq a_{j+1}$. The fraction $p_{j} / q_{j}=\left[a_{0} ; a_{1}, \ldots, a_{j}\right]$, $j \geq 0$, is called a principal convergent of $x$, and $p_{j, k} / q_{j, k}=$ $\left[a_{0} ; a_{1}, \ldots, a_{j}, k\right], j \geq 0,0<k<a_{j+1}$, is called an intermediate converegent of $x$. Note that $p_{j, 0}=p_{j-1}$, $q_{j, 0}=q_{j-1}, p_{j, a_{j+1}}=p_{j+1}, q_{j, a_{j+1}}=q_{j+1}$.

A pair of rational numbers $\frac{a}{m}, \frac{b}{n}$ is called a pair of convergents of $x \in \mathbb{R}$ if $|b m-a n|=1$ and either $\frac{a}{m}<$ $x<\frac{b}{n}$ or $\frac{b}{n}<x<\frac{a}{m}$. It is known that if $\frac{a}{m}, \frac{b}{n}$ is a pair of convergents of $x$, then either $a=p_{j}, m=q_{j}, b=p_{j, k}$, $m=q_{j, k}$ with $j$ even, or $a=p_{j, k}, m=q_{j, k}, b=p_{j}$, $n=q_{j}$ with $j$ odd, and $0<k \leq a_{j+1}$.

Let $I=(-\pi, \pi]$ be a half-open interval, and $\Delta=\Delta_{+} \cup$ $\Delta_{-}$, where $\Delta_{+}=\left\{\left(\theta_{1}, \theta_{2}\right) \in I^{2}: 0<\theta_{1}<\theta_{2}+\pi<\pi\right\}$, $\Delta_{-}=\left\{\left(\theta_{1}, \theta_{2}\right) \in I^{2}: 0<\theta_{2}<\theta_{1}+\pi<\pi\right\}$. Denote by $\llbracket x \rrbracket$ an integer closest to $x \in \mathbb{R}$ such that $-\frac{1}{2}\langle\langle x\rangle:=$ $x-\llbracket x \rrbracket \leq \frac{1}{2}$. Denote a line segment with the endpoints $\zeta_{1}, \zeta_{2} \in \mathbb{C}$ by $\ell\left(\zeta_{1}, \zeta_{2}\right)$, and a triangle with the vertices $\zeta_{1}, \zeta_{2}, \zeta_{3} \in \mathbb{C}$ by $\triangle\left(\zeta_{1}, \zeta_{2}, \zeta_{3}\right)$.

Proposition 1. Let $m, n>0$ be relatively prime integers, and $\theta \in \mathbb{R}$. If $\left(2 \pi\left\langle\frac{m \theta}{2 \pi}\right\rangle, 2 \pi\left\langle\frac{n \theta}{2 \pi}\right\rangle\right) \in \Delta_{+}$, then there exists a unique $0<r<1$ such that for $z=r \mathrm{e}^{\mathrm{i} \theta}$,

$$
\mathcal{T}=\left\{T_{j}=\triangle\left(z^{j+m}, z^{j+n}, z^{j}\right)\right\}_{j \in \mathbb{Z}}
$$

is a triangular spiral multiple tiling of $\mathbb{C}^{*}=\mathbb{C} \backslash\{0\}$ of multiplicity $v=\left(n\left\langle\frac{m \theta}{2 \pi}\right\rangle-m\left\langle\frac{n \theta}{2 \pi}\right\rangle\right) / 2 \pi$.

Proof. If $\left(2 \pi\left\langle\frac{m \theta}{2 \pi}\right\rangle, 2 \pi\left\langle\frac{n \theta}{2 \pi}\right\rangle\right) \in \Delta_{+}, \mathcal{T}$ is a (multiple) tiling if and only if $z^{m+n}$ lands on the line segment $\ell\left(z^{m}, z^{n}\right)$. So $r$ is determined as a root of the equation $f(r)=0$ where

$$
f(r):=r^{m} \sin n \theta-r^{n} \sin m \theta+\sin (m-n) \theta,
$$

by $[14$, Lemma 4$]$. The existence and uniqueness of the root $r \in(0,1)$ follows from the observation that $f(r)$ is a monotone decreasing function of $0 \leq r \leq 1$, such that $f(0)>0>f(1)$.

The integers $m, n>0$ in Proposition 1 are called an $o p$ posed parastichy pair of $\mathcal{T}$. The case $\left(2 \pi\left\langle\frac{m \theta}{2 \pi}\right\rangle, 2 \pi\left\langle\frac{n \theta}{2 \pi}\right\rangle\right) \in$ $\Delta_{-}$shall also have a triangular spiral multiple tiling, which we omit here.

The following Proposition follows from [14, Proposition 3]. Denote the principal argument of $z \in \mathbb{C}$ by $-\pi<\operatorname{Arg}(z) \leq \pi$.

Proposition 2. Let $z=r \mathrm{e}^{\mathrm{i} \theta}, 0<r<1, \theta \in \mathbb{R}$, and $m, n>0$. Suppose that $\operatorname{Arg}\left(z^{n}\right)<0<\operatorname{Arg}\left(z^{m}\right)$, and that (1) is a triangular spiral multiple tiling of multiplicity $v>0$. Then there exist integers $a, b>0$ such that $a / m<$ $b / n$ is a pair of convergents of $x=\theta / 2 v \pi$, and we have $v=\left(n\left\langle\frac{m \theta}{2 \pi}\right\rangle-m\left\langle\frac{n \theta}{2 \pi}\right\rangle\right) / 2 \pi=(b m-a n) / 2 \pi$.

Proposition 3. Let $\theta \in \mathbb{R}$ and $v \in \mathbb{N}$. Suppose that $a / m<b / n$ is a pair of convergents of $\theta / 2 v \pi$, and $m, n>$ 0 are sufficiently large. Then there exists a unique $0<$ $r<1$ such that for $z=r \mathrm{e}^{\mathrm{i} \theta}$, (1) is a triangular spiral multiple tiling of $\mathbb{C}^{*}$ of multiplicity $v$.

Proof. If $m, n$ are large, then $a / m, b / n$ are close to $\theta / 2 v \pi$, and so we have $\left(2 \pi\left\langle\frac{m \theta}{2 \pi}\right\rangle, 2 \pi\left\langle\frac{n \theta}{2 \pi}\right\rangle\right) \in \Delta_{+}$. Proposition 1 is applied to obtain the tiling (1). The multiplicity is obtained from [14, Proposition 3].

\section{Shape limit in triangular spiral tilings}

Let $v>0, \theta \in(-v \pi, v \pi]$. In this section we suppose that $\theta / 2 v \pi$ is a fixed irrational number. In the continued fraction expansion of $x=\theta / 2 v \pi$, we consider the 
sequences $q_{j}$ and $q_{j, k}, j>0,0 \leq k \leq a_{j+1}$, as defined in Sect. 2. For each $j>0$ and $0 \leq k \leq a_{j+1}$, denote by $a_{j, k} / m_{j, k}<b_{j, k} / n_{j, k}$ a pair of convergents of $x=\theta / 2 v \pi$ such that $\left\{m_{j, k}, n_{j, k}\right\}=\left\{q_{j}, q_{j, k}\right\}$. Suppose that $j$ is sufficiently large that $\left(2 \pi\left\langle\frac{m_{j, k} \theta}{2 \pi}\right\rangle, 2 \pi\left\langle\frac{n_{j, k} \theta}{2 \pi}\right\rangle\right) \in \Delta_{+}$. Let $0<r=r_{j, k}<1$ be the root of the Eq. (2), and $z_{j, k}=r_{j, k} \mathrm{e}^{\mathrm{i} \hat{\theta} \theta}$. Then we obtain a (multiple) tiling (1) with an opposed parastichy pair $\{m, n\}=\left\{m_{j, k}, n_{j, k}\right\}$.

Lemma 4. $\operatorname{Arg}\left(z_{j, k}^{q_{j, k}}\right) \rightarrow 0$ as $j \rightarrow \infty$.

Proof. It is known that

$$
\left|\frac{\theta}{2 v \pi}-\frac{p_{j}}{q_{j}}\right| \leq \frac{C}{q_{j}^{2}}
$$

where the constant $C>0$ is independent of $j$. Hence

$$
\begin{gathered}
\left|\operatorname{Arg}\left(z_{j, k}^{q_{j, k}}\right)\right| \leq\left|\operatorname{Arg}\left(z_{j}^{q_{j}}\right)\right|=2 \pi\left|\left\langle\frac{q_{j} \theta}{2 \pi}\right\rangle\right| \\
=2 \pi\left|\frac{q_{j} \theta}{2 \pi}-v p_{j}\right| \leq \frac{2 C v \pi}{q_{j}} \rightarrow 0
\end{gathered}
$$

as $j \rightarrow \infty$.

Lemma 5. Let $v>0, \theta \in(-v \pi, v \pi]$, and suppose that $\theta / 2 v \pi$ is an irrational number. Then, the angles $\angle\left(1, z_{j, k}^{m_{j, k}}, z_{j, k}^{n_{j, k}}\right)$ and $\angle\left(z_{j, k}^{m_{j, k}}, z_{j, k}^{n_{j, k}}, 1\right)$ tend to 0 as $j \rightarrow \infty$.

Proof. By [14, Lemma 4], the four points $z_{j, k}^{m_{j, k}}, 0, z_{j, k}^{n_{j, k}}$, 1 lie on a same circle. Thus we have $\angle\left(1, z_{j, k}^{m_{j, k}}, z_{j, k}^{n_{j, k}}\right)=$ $\angle\left(1,0, z_{j, k}^{n_{j, k}}\right)=\operatorname{Arg}\left(z_{j, k}^{n_{j, k}}\right) \rightarrow 0$ as $j \rightarrow \infty$, and similarly $\angle\left(z_{j, k}^{m_{j, k}}, z_{j, k}^{n_{j, k}}, 1\right)=\angle\left(z_{j, k}^{m_{j, k}}, 0,1\right)=\operatorname{Arg}\left(z_{j, k}^{m_{j, k}}\right) \rightarrow 0$ as $j \rightarrow \infty$.

Lemma 6. Suppose that the coefficients $\left\{a_{j}\right\}_{j \geq 0}$ in the continued fraction expansion $\theta / 2 v \pi=\left[a_{0} ; a_{1}, a_{2}, \ldots\right]$ are bounded. Then we have

$$
0<1-r_{j, k} \leq \frac{C}{m_{j, k}^{3}},
$$

where $C>0$ is a constant independent of $j, k$.

Proof. We shall adopt a notation $\varphi=O\left(\mathrm{~m}^{-s}\right)$ when there exists a constant $C$ independent of $j, k$ such that $|\varphi| \leq C / m_{j, k}^{s}$. Since the coefficients $a_{j}$ are bounded, the ratios $n_{j, k} / m_{j, k}$ are also bounded, so we may write $O\left(m^{-s}\right)=O\left(n^{-s}\right)$. We have

$$
\begin{aligned}
& \sin m \theta=2 \pi\left\langle\frac{m \theta}{2 \pi}\right\rangle-\frac{(2 \pi)^{3}}{6}\left\langle\frac{m \theta}{2 \pi}\right\rangle^{3}+O\left(m^{-5}\right) \\
& =O\left(m^{-1}\right)
\end{aligned}
$$

by $(3)$, and

$$
\begin{aligned}
& \sin n \theta-\sin m \theta+\sin (m-n) \theta \\
& =4 \pi^{3}\left\langle\frac{m \theta}{2 \pi}\right\rangle\left\langle\frac{n \theta}{2 \pi}\right\rangle\left\langle\frac{(m-n) \theta}{2 \pi}\right\rangle+O\left(m^{-5}\right) \\
& =O\left(m^{-3}\right),
\end{aligned}
$$

SO

$$
\frac{\sin (m-n) \theta}{\sin m \theta-\sin n \theta}=1+O\left(m^{-2}\right)
$$

where we denote by $m=m_{j, k}, n=n_{j, k}$ for the sake of simplicity. Since $0<r=r_{j, k}<1$ is a root of (2), we have $\lim _{j \rightarrow \infty} r_{j, k}^{m_{j, k}}=\lim _{j \rightarrow \infty} r_{j, k}^{n_{j, k}}=1$, and

$$
r_{j, k}^{m_{j, k}}=1+O\left(m^{-2}\right), \quad r_{j, k}^{n_{j, k}}=1+O\left(m^{-2}\right) .
$$

Let $t_{j, k}:=1-r_{j, k}$. Then we have $t_{j, k}=O\left(m^{-3}\right)$ by (4), which completes the proof.

Suppose that $\theta / 2 v \pi$ is a quadratic irrational. Then it has a periodic continued fraction expansion

$$
\begin{aligned}
& \frac{\theta}{2 v \pi}=\left[a_{0} ; a_{1}, a_{2}, \ldots\right] \\
& \quad=\left[a_{0} ; a_{1}, \ldots, a_{j_{0}}, \overline{b_{1}, \ldots, b_{d}}\right] \\
& \quad=\left[a_{0} ; a_{1}, \ldots, a_{j_{0}}, b_{1}, \ldots, b_{d}, b_{1}, \ldots, b_{d}, \ldots\right] .
\end{aligned}
$$

We may assume that $j_{0}, d$ are even, by choosing larger ones if necessary. For each $1 \leq h \leq d$, let

$$
\omega_{h}=\left[b_{h} ; \overline{b_{h+1}, \ldots, b_{d}, b_{1}, \ldots, b_{h}}\right]
$$

be a purely periodic continued fraction.

Let $R(\theta, v)$ be the set of ratios $\left(z_{j, k}^{n_{j, k}}-1\right) /\left(z_{j, k}^{m_{j, k}}-1\right)$ for $j>0$ and $0<k \leq a_{j}$. Let

$$
\Omega(\theta, v):=\Omega(R(\theta, v))
$$

be the limit set, i.e., the set of the accumulation points, of $R(\theta, v)$.

Theorem 7. Suppose that $\theta / 2 v \pi$ is a quadratic irrational. Then we have

$$
\Omega(\theta, v)=\left\{\left(\omega_{j}-k\right)^{(-1)^{j}}: 0<j \leq d, 0<k \leq b_{j}\right\} .
$$

In particular, it is a finite set of quadratic irrationals.

Proof. Since $\theta / 2 v \pi$ is a quadratic irrational, there exists a constant $C_{1}, C_{2}>0$, independent of $j>0,0<k \leq a_{j+1}$, such that

$$
\frac{C_{1}}{q_{j, k}^{2}}<\left|\frac{p_{j, k}}{q_{j, k}}-\frac{\theta}{2 v \pi}\right|<\frac{C_{2}}{q_{j, k}^{2}} .
$$

This implies that

$$
\frac{C_{1}}{m_{j, k}}<\left|\left\langle\frac{m_{j, k} \theta}{2 v \pi}\right\rangle\right|<\frac{C_{2}}{m_{j, k}} .
$$

We have

$$
\begin{aligned}
& \frac{z_{j, k}^{n_{j, k}}-1}{z_{j, k}^{m_{j, k}}-1}=\frac{-1+r^{n} \cos n \theta+\mathrm{i} r^{n} \sin n \theta}{-1+r^{m} \cos m \theta+\mathrm{i} r^{m} \sin m \theta} \\
& =\frac{-1+1+O\left(m^{-2}\right)+\mathrm{i}\left(2 \pi\left\langle\frac{n \theta}{2 \pi}\right\rangle+O\left(m^{-2}\right)\right)}{-1+1+O\left(m^{-2}\right)+\mathrm{i}\left(2 \pi\left\langle\frac{m \theta}{2 \pi}\right\rangle+O\left(m^{-2}\right)\right)} \\
& =\frac{2 \pi \mathrm{i}\left\langle\frac{n \theta}{2 \pi}\right\rangle+O\left(m^{-2}\right)}{2 \pi \mathrm{i}\left\langle\frac{m \theta}{2 \pi}\right\rangle+O\left(m^{-2}\right)} \\
& =\frac{2 \pi \mathrm{i}\left\langle\frac{n \theta}{2 \pi}\right\rangle\left(1+O\left(m^{-1}\right)\right)}{2 \pi \mathrm{i}\left\langle\frac{m \theta}{2 \pi}\right\rangle\left(1+O\left(m^{-1}\right)\right)} \\
& =\frac{\left\langle\frac{n \theta}{2 \pi}\right\rangle}{\left\langle\frac{m \theta}{2 \pi}\right\rangle}\left(1+O\left(m^{-1}\right)\right),
\end{aligned}
$$


where we denote by $m=m_{j, k}, n=n_{j, k}$. Thus it is written as

$$
\frac{z_{j, k}^{n_{j, k}}-1}{z_{j, k}^{m_{j, k}}-1}=\left(\frac{\left\langle\frac{q_{j, k} \theta}{2 v \pi}\right\rangle}{\left\langle\frac{q_{j} \theta}{2 v \pi}\right\rangle}\right)^{(-1)^{j}}\left(1+O\left(q^{-1}\right)\right) .
$$

By using the continued fractions, we have

$$
\begin{aligned}
& \left\langle\frac{q_{j, k} \theta}{2 v \pi}\right\rangle /\left\langle\frac{q_{j} \theta}{2 v \pi}\right\rangle=-\left[a_{j+1}-k, a_{j+2}, a_{j+3}, \ldots\right] \\
& \quad=-\left(\omega_{j}-k\right)
\end{aligned}
$$

for $j$ sufficiently large, and $0 \leq k \leq a_{j+1}$. Thus we obtain Eq. (5).

It is known that most spirals in plant phyllotaxis have divergence angles $\theta$ belonging to a class that might be called 'ultimately golden', or written as $\theta / 2 \pi=$ $\left[a_{0} ; a_{1}, a_{2}, \ldots\right]$ where $a_{j}=1$ for sufficiently large $j[5]$. This is equivalent to the existence of $a, b, c, d \in \mathbb{Z}$, such that $\theta / 2 \pi=(a \tau+b) /(c \tau+d)$ and $a d-b c=1$.

Corollary 8. Suppose that $\theta / 2 v \pi$ has a continued fraction expansion $\theta / 2 v \pi=\left[a_{0} ; a_{1}, a_{2}, \ldots\right]$ such that $a_{j}=1$ for sufficiently large $j$. Then $\Omega(\theta, v)=\{-\tau,-1 / \tau\}$.

Proof. The golden section has a purely periodic continued fraction expansion $\tau=[1 ; 1, \ldots]=[1 ; \overline{1}, 1]$, and

$$
\left\langle\frac{q_{j, 1} \theta}{2 v \pi}\right\rangle /\left\langle\frac{q_{j} \theta}{2 v \pi}\right\rangle=-[0 ; 1,1, \ldots]=-\frac{1}{\tau}
$$

for any $j$.

Figure 4 shows a triangular spiral tiling generated by $z=r \mathrm{e}^{2 \pi \mathrm{i} \tau}, r=0.9965, \tau=(1+\sqrt{5}) / 2$, with an opposed parastichy pair $\{8,13\}$, and the ratio $\left(z^{8}-1\right) /$ $\left(z^{13}-1\right)=-1.348+0.857 \mathrm{i}$. If we fix the divergence angle $2 \pi \tau$ and consider larger Fibonacci numbers as an opposed parastichy pair, for example $\{55,89\}$, then we have $r=0.999989$, and so the ratio $\left(z^{55}-1\right) /\left(z^{89}-1\right)=$ $-1.61208+0.13355 \mathrm{i}$ gets closer to $-\tau=-1.618$.

\section{Acknowledgments}

The authors would like to thank the referee for helpful comments. This work was partially supported by JSPS Kakenhi Grant number 24654029 and Ryukoku University Science and Technology Fund.

\section{References}

[1] R.V. Jean, Phyllotaxis: a systemic study in plant morphogenesis, Cambridge University Press, 1994.

[2] A.V. Shubnikov, Crystal Symmetries, Shubnikov Centennial Papers, Eds.: I. Hargittai, B.K. Vainshtein, Pergamon, Oxford 1988.

[3] Symmetry in Plants, Eds.: R.V. Jean, D. Barabe, World Scientific, Singapore 1998.

[4] I. Adler, Solving the riddle of phyllotaxis, why the Fibonacci numbers and the golden ratio occur on plants, World Scientific, Singapore 2012.

[5] H.S.M. Coxeter, J. Algebra 20, 167 (1972).

[6] N. Rivier, R. Occelli, J. Pantaloni, A. Lissowski, J. Phys. 45, 49 (1984).

[7] L.S. Levitov, Phys. Rev. Lett. 66, 224 (1991).

[8] S. Douady, Y. Couder, J. Theor. Biol. 178, 255 (1996); ibid., 275; ibid., 295.

[9] C. Nisoli, N.M. Gabor, P.E. Lammert, J.D. Maynard, V.H. Crespi, Phys. Rev. Lett. 102, 186103 (2009).

[10] K. van Berkel, R.J. de Boer, B. Scheres, K. ten Tusscher, Development 140, 2253 (2013).

[11] F. Rothen, A.J. Koch, J. Phys. (France) 50, 633 (1989).

[12] S. Hotton, V. Johnson, J. Wilbarger, K. Zwieniecki, P. Atela, C. Gole, J. Dumais, J. Plant Growth Regulation 25, 313 (2006).

[13] P. Atela, Math. Model. Nat. Phenom. 6, 173 (2011).

[14] T. Sushida, A. Hizume, Y. Yamagishi, J. Phys. A: Math. Theor. 45, 235203 (2012), (see also the cover page).

[15] A. Hizume, Y. Yamagishi, J. Phys. A: Math. Theor. 44, 015202 (2011) (see also the cover page).

[16] A.Ya. Khinchin, Continued fractions, The University of Chicago Press, Chicago 1964.

[17] G.H. Hardy, E.M. Wright, An introduction to the theory of numbers, 6th ed., Oxford University Press, Oxford 2008. 